\title{
Study of polycaprolactone wet electrospinning process
}

\author{
E. Kostakova ${ }^{1}$, M. Seps ${ }^{2}$, P. Pokorny ${ }^{1}$, D. Lukas ${ }^{1}$ \\ ${ }^{1}$ Department of Nonwovens and Nanofibrous Materials, Faculty of Textile Engineering, Technical University of Liberec, \\ Studentska 2, 46117 Liberec 1, Czech Republic \\ ${ }^{2}$ Institute of Novel Technologies and Applied Informatics, Faculty of Mechatronics, Informatics and Interdisciplinary \\ Studies, Technical University of Liberec, Studentska 2, 46117 Liberec, Czech Republic
}

Received 6 February 2014; accepted in revised form 12 April 2014

\begin{abstract}
Wet electrospinning is a useful method for 3-dimensional structure control of nanofibrous materials. This innovative technology uses a liquid collector instead of the metal one commonly used for standard electrospinning. The article compares the internal structural features of polycaprolactone (PCL) nanofibrous materials prepared by both technologies. We analyze the influence of different water/ethanol compositions used as a liquid collector on the morphology of the resultant polycaprolactone nanofibrous materials. Scanning electron micro-photographs have revealed a bimodal structure in the wet electrospun materials composed of micro and nanofibers uniformly distributed across the sample bulk. We have shown that the full-faced, twofold fiber distribution is due to the solvent composition and is induced and enhanced by increasing the ethanol weight ratio. Moreover, the comparison of fibrous layers morphology obtained by wet and dry spinning have revealed that beads that frequently appeared in dry spun materials are created by Plateau-Rayleigh instability of the fraction of thicker fibers. Theoretical conditions for spontaneous and complete immersion of cylindrical fibers into a liquid collector are also derived here.
\end{abstract}

Keywords: nanomaterials, wet electrospinning, biodegradable polymers, polycaprolactone, liquid collector

\section{Introduction}

Wet electrospinning is a variant of the well-known electrospinning technology. The modification is based on the usage of a liquid collector instead of a solid, mostly metallic one. Wet electrospinning was first introduced in [1,2] as a method for the lab-scale production of nanofibrous scaffolds for tissue engineering. To produce bulky and fluffy material using the common electrospinning method is a tricky matter. This task could be achieved either using special collectors [3] or by the usage of porogen particles, that is, chemical blowing agents, imbedded in between the nanofibers [4]. Wet electrospinning is a relatively simple and effective method to produce three dimensional (sponge-like) materials without sophisticated devices and without special chemical additives. The main application of such bulky materials is predominant in tissue engineering. Materials produced by wet-electrospinning are poly(glycolic acid) [5], chitin [6], cellulose [7], poly(trimethylenecarbonate-co- $\varepsilon$-caprolactone)-block-poly-(p-dioxanone) [8], copolymer of poly(glycolic acid) and poly(lactic acid) and blend of polycaprolactone and collagen or polycaprolactone and elastine [9]. A detailed study devoted to the immersion precipitation process in the electrospinning of polyacrylonitrile (PAN) dissolved in N,N-dimethyl-formamide (DMF) was developed by Fang et al. [10]. The Fang group demonstrated that using a non-solvent as a coagulant, ethanol in this case, polymer solution jets of PAN-DMF can be solidified rapidly resulting in instantaneously 'frozen' solid structures that can then

\footnotetext{
${ }^{*}$ Corresponding author, e-mail: eva.kostakova@tul.cz

(C) BME-PT
} 
be observed using scanning electron microscopy (SEM). The research confirms the important effect of the liquid collector bath height, the collecting distance, the electric field intensity and the concentration of the original polymer solution on the final morphology. We have used the Fang group method to study the evolution of PCL fiber morphology during wet electrospinning.

The immersion precipitation is a widely used method of classic fiber spinning technologies. In this respect, the wet electrospinning is a variant of the classic wet fiber spinning method. In this process the spinneret is submerged in a chemical bath that is a nonsolvent for the polymer but which is compatible with the used solvent of the polymer [11]. Compared to the classic wet spinning process, wherein the nozzles are directly immersed into a coagulation bath, a small air gap (usually about $10 \mathrm{~mm}$ ) is between the nozzle and the coagulation bath in the dry-jet-wet spinning process [12]. The air gap prevents polymer precipitation inside the spinnerets. The formation of a 'skin' of coagulated polymer occurs on first contact between a polymer jet filament and the coagulation bath [13]. The 'skin' clearly prevents the fiber from breaking up into spherical drops due to PlateauRayleigh instability [14]. This instability usually appears in the electrospinning of extremely dilute polymer solutions, where the resultant droplet formations are described as a 'pearl effect' on the fibers, 'beads-on-a-string', or simply as 'polymer beads' [15].
This study focuses on PCL (dissolved in chloroform/ ethanol) wet electrospinning with water/ethanol mixtures employed as the liquid collector. The influence of the liquid collector composition on the final electrospun fibrous structure is studied here. The experiments are accompanied by theoretical analysis to suggest the general conditions needed for the production of bulky fibrous materials by wet electrospinning technology. The bulky, biodegradable, fibrous materials are of great interest for use as scaffolds for tissue engineering and this article provides a detailed study of the wet electrospinning process leading to their laboratory manufacture.

\section{Materials and methods}

Poly- $\varepsilon$-caprolactone (PCL), having an average molecular weight of $42500\left[M_{\mathrm{n}}\right]$, density $1.145 \mathrm{~g} \cdot \mathrm{cm}^{-3}$, melting point $60^{\circ} \mathrm{C}$ from Sigma Aldrich Inc. and dissolved in a chloroform/ethanol mixture (9:1 by weight), is mixed for 24 hours by a magnetic stirrer. The polymer concentration in the solution is $15 \mathrm{wt} \%$. The solution of PCL in pure chloroform is used to evaluate the influence of the ethanol in the three component PCL solution. The dish of the liquid collector used for wet electrospinning is filled with a mixture of distilled water and ethanol at various volume fractions $(10: 0 ; 9: 1 ; 8: 2 ; 7: 3 ; 6: 4 ; 5: 5 ; 4: 6$; $3: 7 ; 2: 8 ; 1: 9 ; 0: 10)$.

The electrospinning apparatus used for wet electrospinning experiments (Figure 1) consists of a syringe equipped with a metal hypodermic needle of inter-

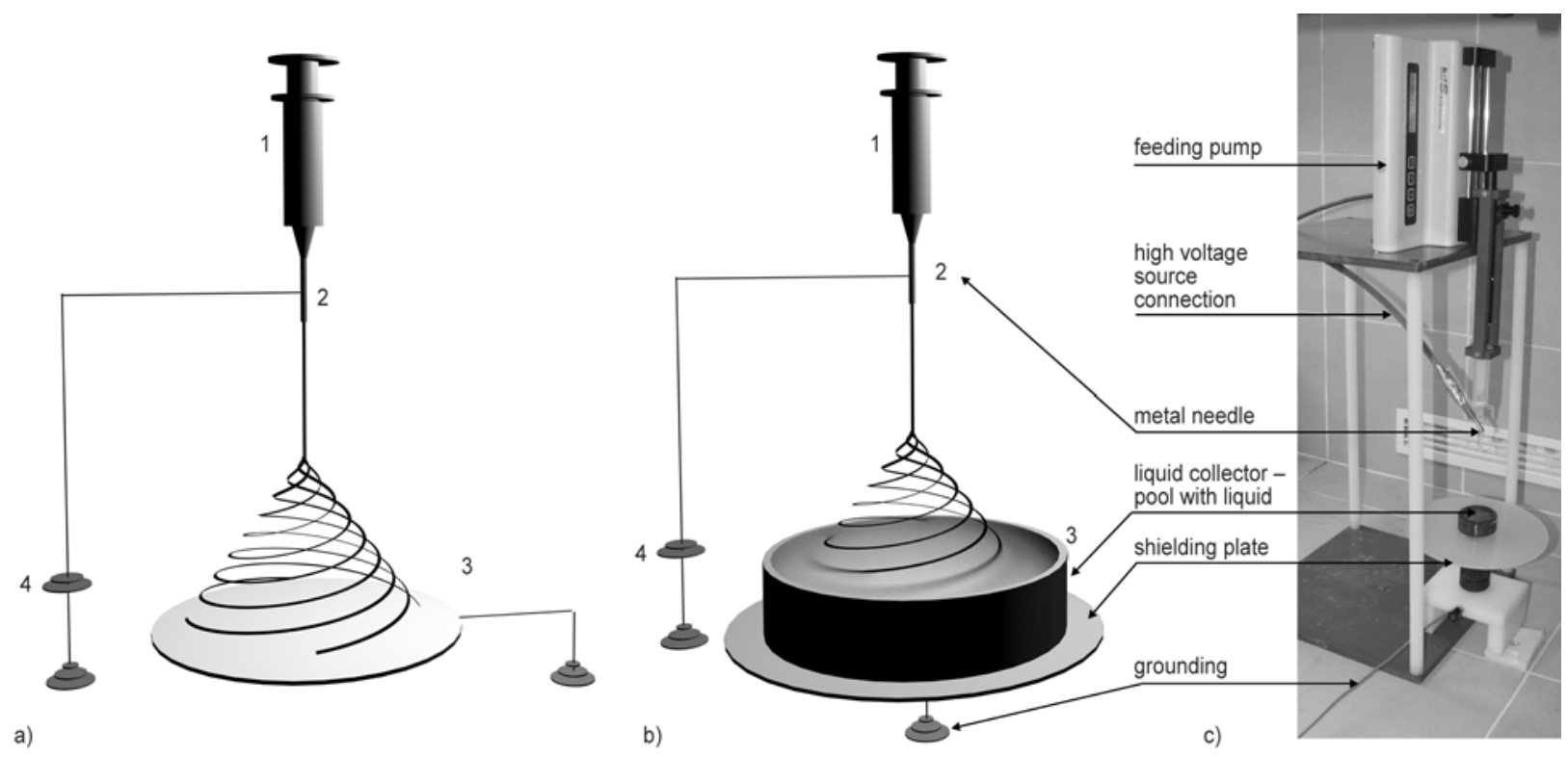

Figure 1. A schematic diagram and a photo of the standard dry needle electrospinning apparatus (a), wet electrospinning (b) and a photo of the device used in the experiments (c). The diagram shows the syringe connected to the feeding pump (1); metal hypodermic needle (2); metallic or liquid collector (3) and grounded high voltage source (4). 
nal diameter $0.9 \mathrm{~mm}$ and length $33 \mathrm{~mm}$. The needle is connected to the positive pole of a high voltage source (Spellman SL 150) and placed in the feeding device (KDScientific KDS-100). A grounded metallic plate is placed inside the dish/pool filled with the collector liquid. A shielding plate made of polyvinyl chloride (PVC) was used to focus a jet into the liquid collector. The process parameters are: positive voltage of $20 \mathrm{kV}$ at the needle; the distance between needle end and the liquid collector surface is adjusted to $100 \mathrm{~mm}$; pool depth $10 \mathrm{~mm}$; pool inner diameter $40 \mathrm{~mm}$; feeding velocity $7 \mathrm{~mL} \cdot \mathrm{h}^{-1}$. Each sample was prepared from $0.5 \mathrm{~mL}$ of PCL solution, thus the final average area mass density of the electrospun material is about $68 \mathrm{~g} \cdot \mathrm{m}^{-2}$. The wet electrospun samples were dried at a laboratory temperature of $20^{\circ} \mathrm{C}$ and a relative air humidity of $60 \pm 5 \% \mathrm{RH}$.

The surface tension (bubble tension meter Krüss PocketDyne) and the conductivity (WTW Inolab GnInd 720) of the collector liquid were measured. Samples structures were analyzed using scanning electron microscopy (FEI Company - Phenom) and image analyzer software (Lucia G 4.82, LIM). Crosssectional micro-photographs were obtained from samples broken at low temperature in liquid Nitrogen.

\section{Results and discussion}

The results presented here are divided into two main parts. The first part describes the fiber morphologies obtained using various conditions for the PCL wet electrospinning process. The last part focuses on the influence of the liquid collector on the electrospinning process and the material structure, that is, the creation of nanofibers, microfibers and beads in various combinations. These morphological transmutations are closely related with the varying conditions under which the wet electrospinning processes were run. Explanations of the causes of the transmutations are discussed thereafter.

\subsection{Fiber morphology}

The wet electrospun samples had a similar total surface density, about $60 \mathrm{~g} \cdot \mathrm{m}^{-2}$, and dry thickness of $500 \pm 20 \mu \mathrm{m}$. The surface mass density was smaller than expected bulk one, because some fibers were able to stay at the edges of the pool or did not fly directly onto the liquid collector surface. The scanning electron microscope images reveal an inner structure composed of fibers belonging to two groups of distinct diameters. Figure 2a shows representative sample morphology produced using the liquid collector consisting of $90 \mathrm{wt} \%$ of ethanol. In this case, the diameters of the nanofibers group range from 95 up to $1000 \mathrm{~nm}$, while the diameters of the microfiber fraction range between $2700-5000 \mathrm{~nm}$ as can be seen in Figure 3. The average diameter of the nanofibers group is $239 \pm 94 \mathrm{~nm}$, while the average diameter of the microfiber fraction is $3664 \pm 731 \mathrm{~nm}$, see Figure 2a, 2b and Figure 3. Diameters of fibers electrospun onto the metal plate collector was measured for comparison. Their average diameter is $162 \pm 62 \mathrm{~nm}$. This dry electrospun material consists of nanofibers that only interconnect the spherical particles/beads having an average equivalent diameter of $14.6 \pm 4.2 \mu \mathrm{m}$, see Figure 2c. A microfiber fraction without any nanofibrous element is generated when pure chloroform is used as a solvent and wet electrospinning is performed into the water/ethanol (1:9) liquid collector. The fiber diameter of this wet electropun PCL material is $2.0 \pm 0.6 \mu \mathrm{m}$, as shown in Figure $2 \mathrm{~d}$. Thanks to the wet electrospinning method, microfibers are solidified in the liquid of the collector (see Figure $2 \mathrm{a}$ and $2 \mathrm{~b}$ ). Therefore the PlateauRayleigh instability does not develop in this case unlike in the sample created from the same PCL solution by classic electrospinning that uses the metal plate collector. In the latter, originally created microfibers are detached from the spherical polymer bodies interconnected by nanofibers only (see Figure 2c). When electrospinning onto the water surface took longer than in the previously described experiments, that is, more than $0.5 \mathrm{~mL}$ of solution is delivered, then special structures consisting of a 'frozen' microfibers and drops developed from them were found (Figure 4).

The twofold structure of the fibers, as depicted in Figure $2 \mathrm{a}$ and $2 \mathrm{~b}$, is clearly caused by the two-component solvent (chloroform/ethanol). Chloroform is a good solvent for PCL, but ethanol is not. The solvent composition influences the final fiber structure. This statement results from the fact that the wet electrospun PCL material from chloroform solution only, see Figure 2d, does not generate the dual structure, that is, it is composed of fibers of nearly uniform diameters. The structure of the wet electrospun PCL from the chloroform/ethanol solution into the mixture of water/ethanol results in a dual structured material composed of micro and nanofibers. Conversely, classically electrospun chloroform/ethanol solution 

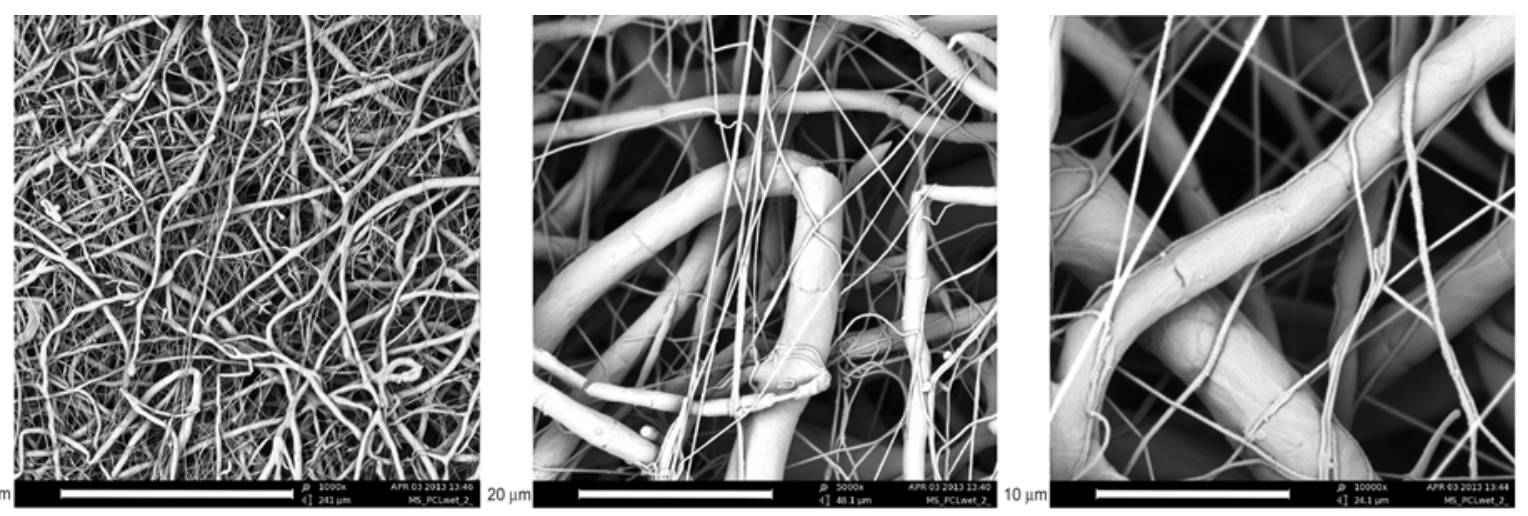

a)
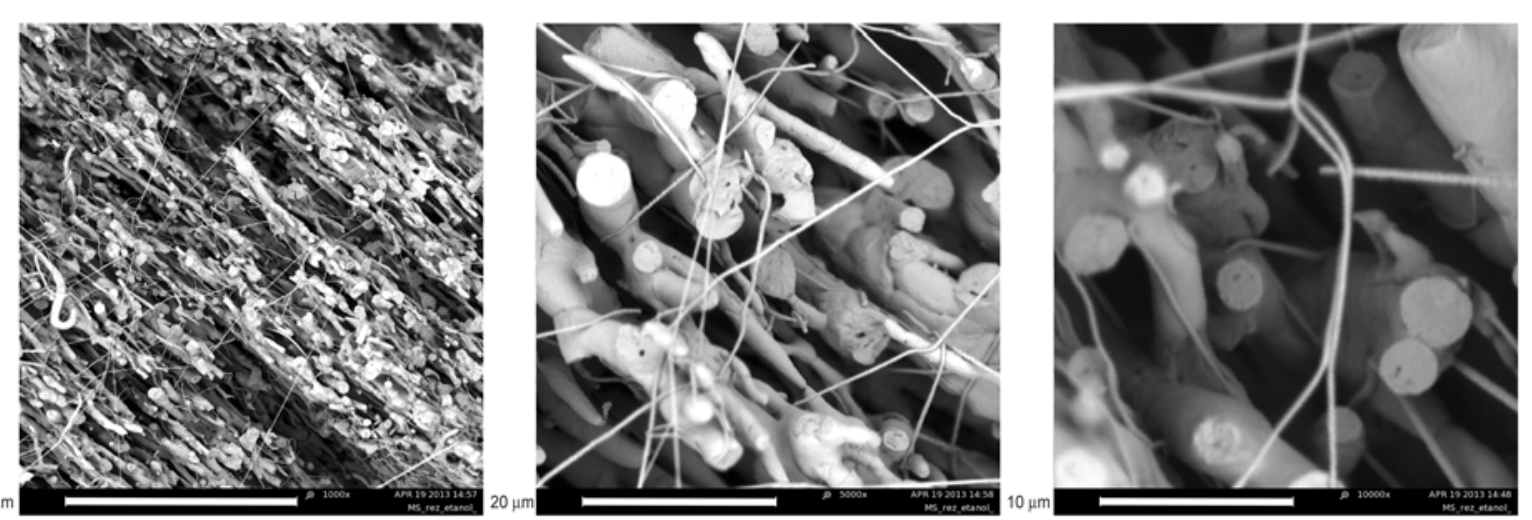

b)
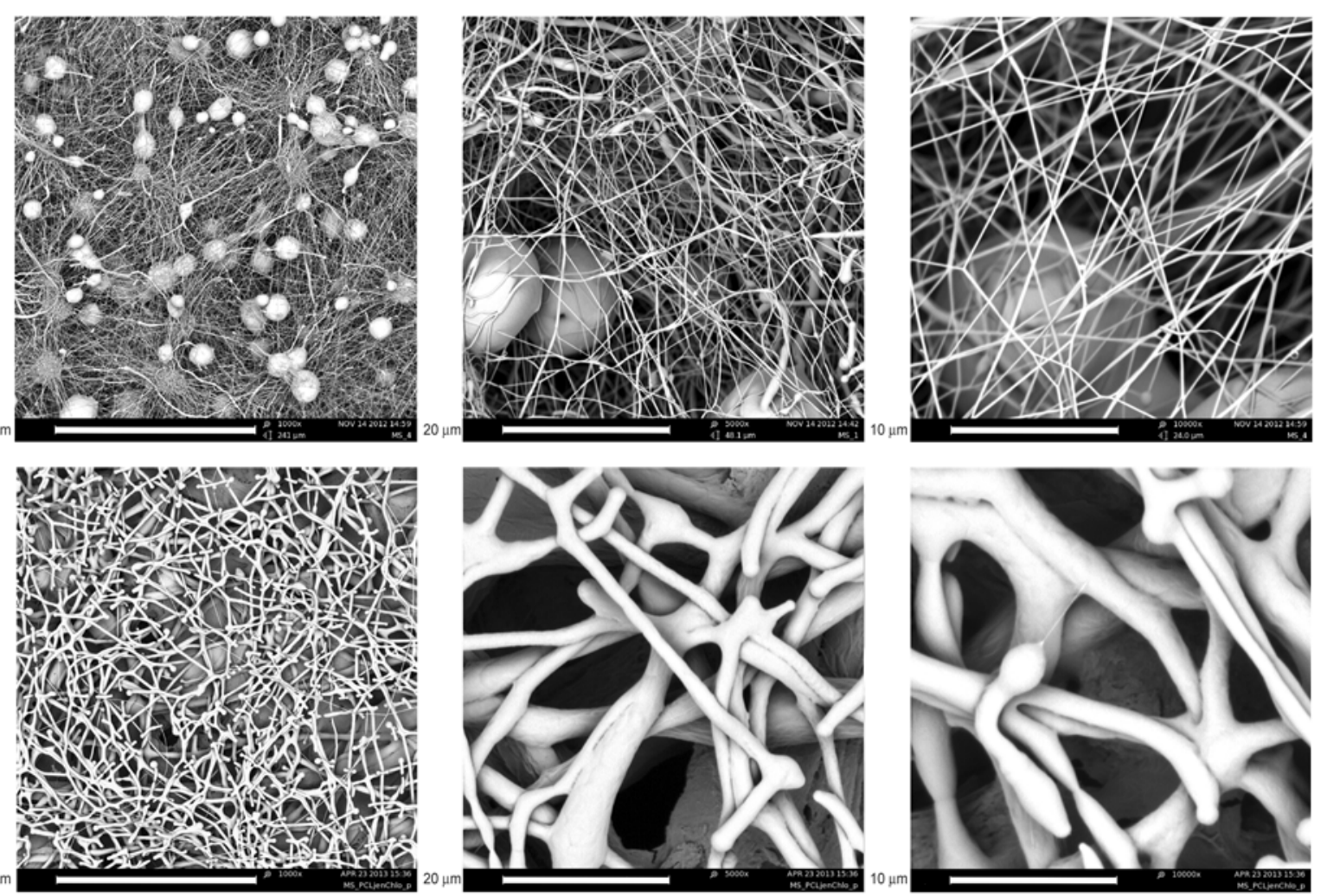

Figure 2. Scanning electron images of electrospun materials - three images in line with different magnification for each of these materials: (a) PCL fibers (from chloroform/ethanol 9:1 solution) wet electrospun into water/ethanol (1:9) liquid collector; (b) cross-sectional view of the same sample - PCL fibers (from chloroform/ethanol 9:1 solution) wet electrospun into water/ethanol (1:9) liquid collector; (c) PCL material electrospun from chloroform/ethanol 9:1 solution onto the metal plate collector; (d) PCL nanofibrous layer electrospun from pure chloroform solution into water/ethanol (1:9) liquid collector. The scale is $120 \mu \mathrm{m}$ on the left, $20 \mu \mathrm{m}$ in the middle and $10 \mu \mathrm{m}$ on the right side. 


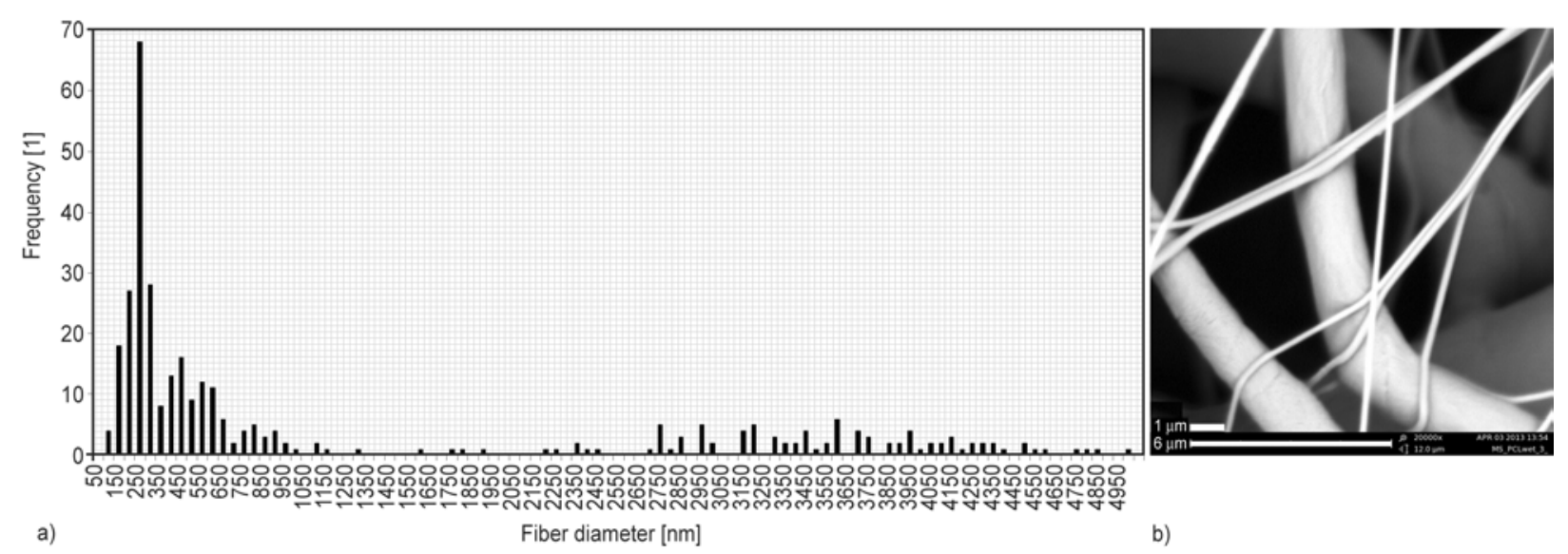

Figure 3. Histogram of fiber diameters in the PCL fibrous material electrospun into the liquid collector (water/ethanol weight ratio 1:9) (a), and SEM image representing dual structure of the wet electrospun material (scale bars represent 1 and $6 \mu \mathrm{m}$ ) is put in to proof the nanofibers presence in the sample (b).

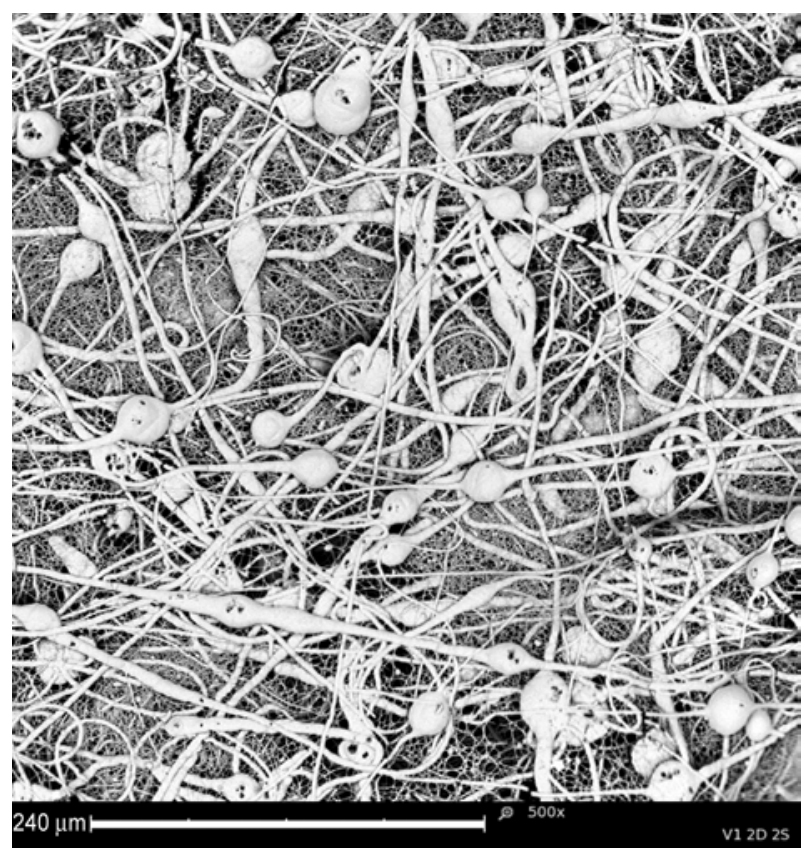

Figure 4. Scanning electron images of electrospun material: PCL fibers (from chloroform/ethanol 9:1 solution) wet electrospun into distilled water only. $2.5 \mathrm{~mL}$ of PCL polymer solution were used, which is five times more than for the production of other samples described here. The scale is $240 \mu \mathrm{m}$.

of PCL provides beads on strings that are collected on a metallic collector. Thus one can conclude that the dual structure with micro and nanofibers is created in the spinning space between the spinning electrode and the collector, where whipping and branching of the polymer liquid jet occurs. Jet branching during the electrospinning process was described for various polymer solutions including PCL in the work [8]. The creation of beads in dry electrospun samples is the consequence of the PlateauRayleigh instability of a thicker fraction of fibers trapped on the collector. Probably, nanofibers do not undergo the Plateau-Rayleigh instability thanks to the higher degree of their cohesion compared with the much thicker microfibers that contain more solution. The characteristic hydrodynamic time for the fiber-bead transition is evidently longer than the flight time of a jet element inside the spinning space.

More detailed analysis shows (see Figure 5) that the fraction of nanofibers decreases with an increasing concentration of water in the liquid collector bath. These changes of the material's morphology are the consequence of increased surface tension of the liquid collector caused by the higher water ratio. The higher surface tension obstructs fibers from being immersed into the collector liquid and hence the collected fibers are not completely submerged. The fibers trapped on the liquid collector surface are in contact with other nascent fibers and it leads to a compact material structure with smaller pores and smaller fibers absorbed by bigger ones before their complete solidification. These changes are reflected in the transition from the three-dimensional structures with high porosity to a compact structure with lower porosity.

\subsection{The effect of changing the liquid collector}

Fibers were immersed fully into the water/ethanol bath only in the case of the high ethanol concentration that is, $2: 8 ; 1: 9$ and $0: 10$ of weight ratios. In these cases a three-dimensional fibrous structure is created in the bulk of the liquid collector. Lower ethanol concentrations cause fibers to collect on the liquid surface as can be seen in Figure 6. The reason for this phenomenon is the increasing value of the col- 

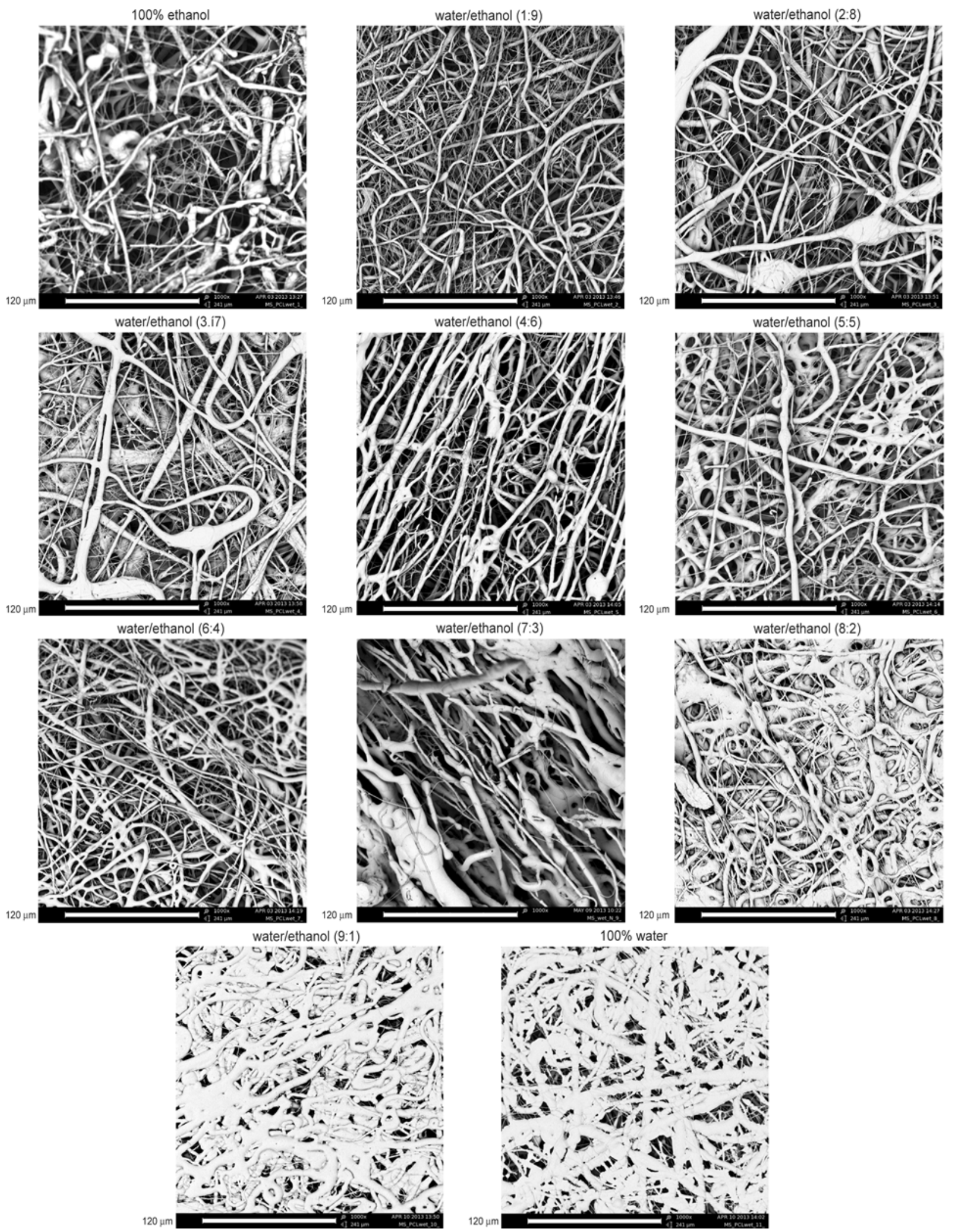

Figure 5. Scanning electron images of electrospun materials: PCL fibers (from chloroform/ethanol 9:1 solution) wet electrospun into different liquid collectors described above each image. Scale bar represents $120 \mu \mathrm{m}$.

lector liquid surface tension with the water ratio. Theoretical analysis of this phenomenon is introduced in paragraph 3.3.
The existence of the transition point when immersing PCL fibers into the collector liquid depicted in Figure 6 is supplemented by observing convention- 


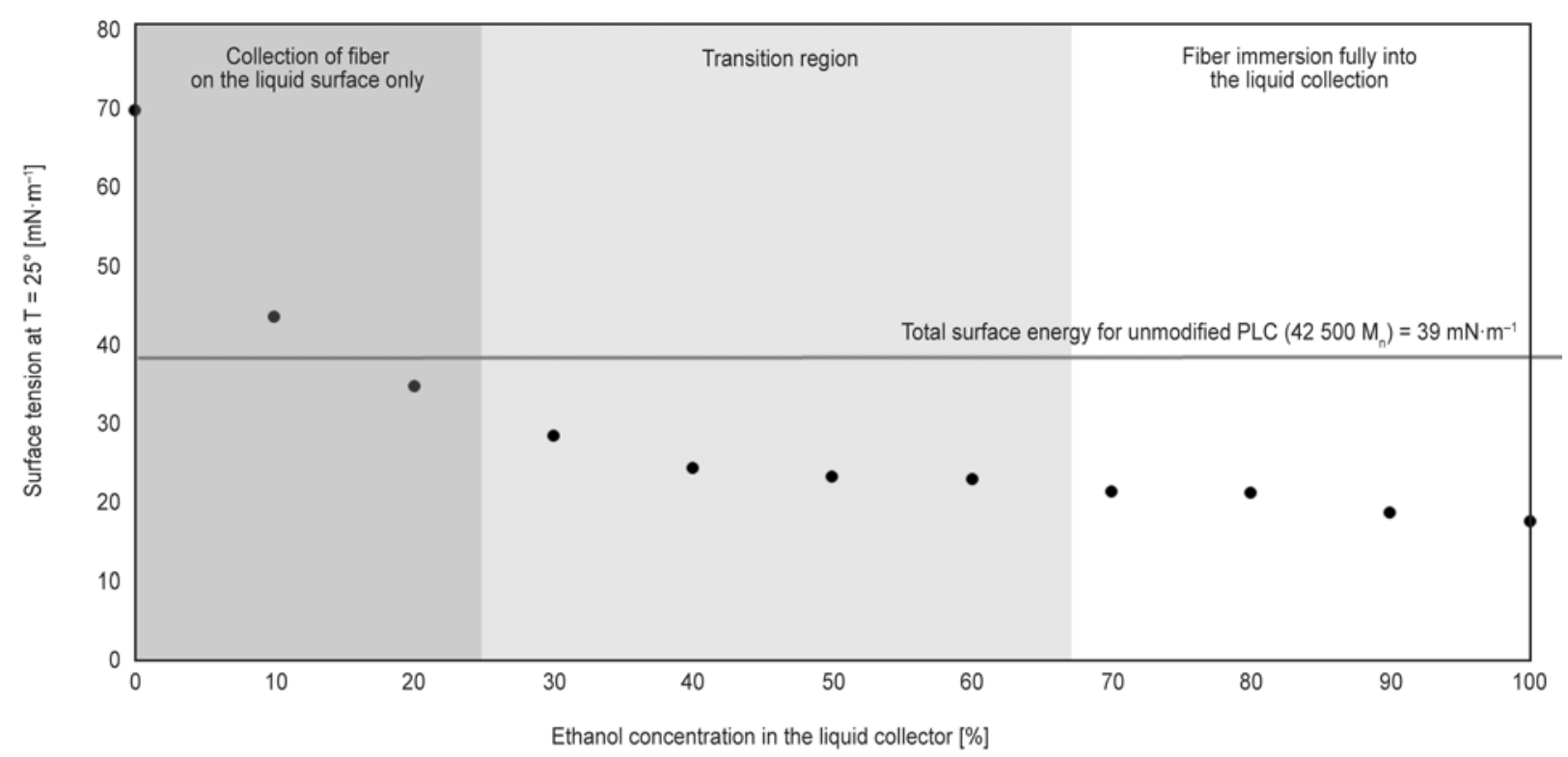

Figure 6. Graphical representation of the effect on fiber behavior of liquid collector composition: The horizontal line close to $40 \mathrm{mN} \cdot \mathrm{m}^{-1}$ represents the transition region above which some fibers remain laying on the liquid collector level and only part of them are dipped in the liquid. Below this transition line all fibers are submerged in the liquid. Surface tension measurements of the liquid collector are carried out at temperature of $25^{\circ} \mathrm{C}$. Standard deviations are below $0.2 \mathrm{mN} \cdot \mathrm{m}^{-1}$. Each measurement was repeated four times. The gray line in the graph represents the surface tension of PCL measured with the same PCL by Yildirim [16].

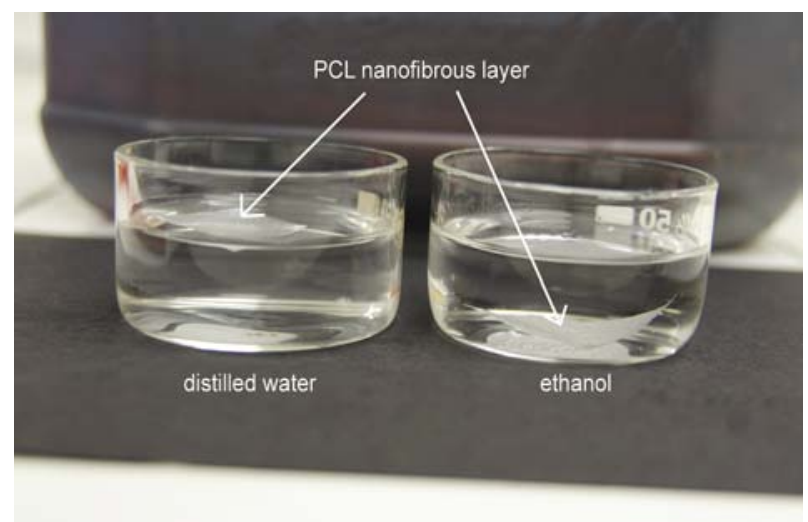

Figure 7. The photo of dry electrospun PCL layer having the surface density of $6 \mathrm{~g} \cdot \mathrm{m}^{-2}$ inserted into the distilled water (left side) and ethanol (right side) ally electrospun PCL material after its being applied to distilled water and ethanol. The layer of nanofibers floats on the distilled water surface whereas it sinks into the ethanol bath as depicted in Figure 7. The electrical conductivity of the liquids used as a collector slowly decreases with increasing ethanol concentration in the PCL solution as is visible in Figure 8 . Both the electrical conductivity of the used collector liquid and the collecting distance are important in the process of wet electrospinning. The distance between the end of needle spinning electrode and the level of the liquid collector affect the electric field strength inside the spinning space as has been described in detail by Fang et al. [10].

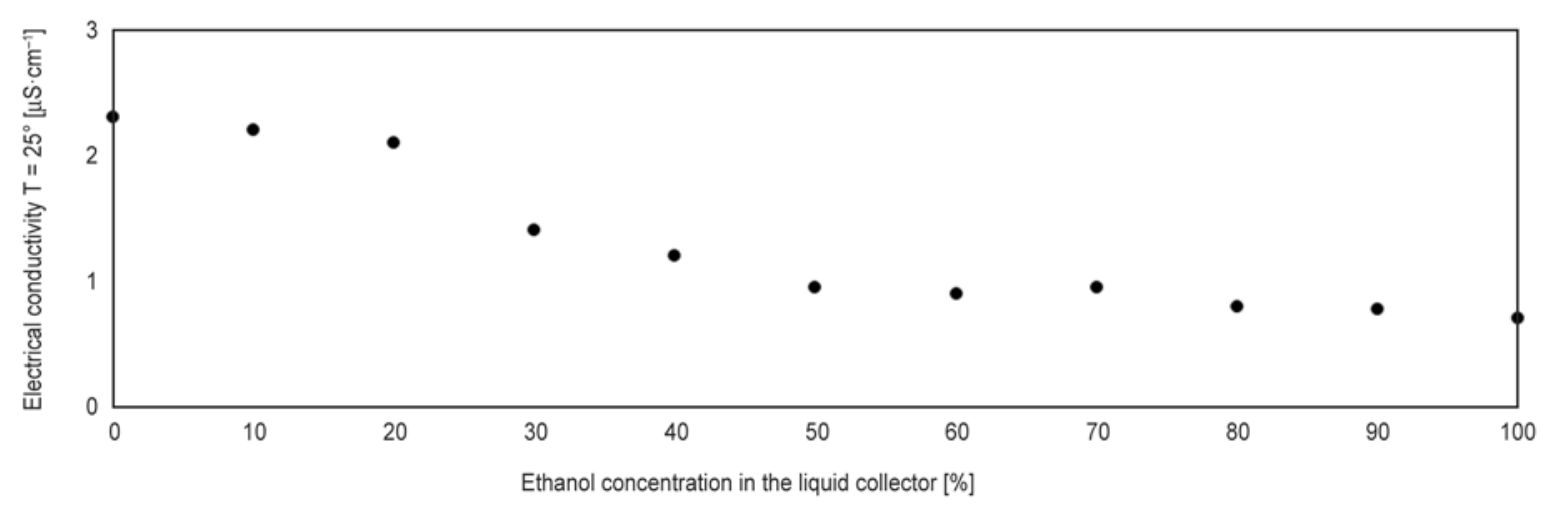

Figure 8. The collector liquid electrical conductivity depends on the ethanol concentration. The measurements were carried out at $25^{\circ} \mathrm{C}$. Each measurement was repeated four times. Standard deviations are below $0.1 \mu \mathrm{S} \cdot \mathrm{cm}^{-1}$. 
Based on the images from the scanning electron microscope (Figure 2a, 2b and Figure 5), the 9:1 water/ethanol mixture with a conductivity of $1.2 \mu \mathrm{S} \mathrm{cm}^{-1}$ and a surface tension of $26 \mathrm{mN} \cdot \mathrm{m}^{-1}$ was chosen as the most appropriate liquid collector composition. Materials obtained using this kind of collector liquid have a fibrous three-dimensional structure with high porosity and almost no defects such as attached fibers or beads.

\subsection{Lift force on a partly submerged cylinder}

Efforts to clarify the problem of fiber mass immersion into a collector liquid during wet electrospinning led to the following theoretical explanation. The condition for spontaneous and complete immersion of a cylindrical fiber (cylinder) lying on a liquid surface are related to the study of water-spiders moving on the water's surface [17].

A lift force acting on a partly submerged cylinder in a liquid in rest is composed of two components. The first is the surface tension force $f_{\mathrm{T}}$, while the second is called the pressure force $f_{\mathrm{P}}$. Both these forces were introduced by Keller [18]. The nature of $f_{\mathrm{T}}$ and $f_{\mathrm{P}}$ is derived from the mechanical analysis of the cross-sectional geometry of the partly submerged cylinder depicted in Figure 9. The angle $\beta$ that exists between a vertical line passing the cylinder axes and a line connecting the axis with a contact line is considered here in the interval $(0, \pi / 2)$. The deformed liquid surface, due to the cylinder weight and the liquid surface tension $\gamma$, together with the immersed part of the cylinder forms a shape resembling a ship's hull below its waterline. The hull volume can be split into three departments subdivided by vertical walls. Two wing departments, denoted as 1 and 3, occupy a space equal to the liquid displaced by the meniscus up to the vertical walls containing the contact line of a liquid, gas and solid. The central department 2 is

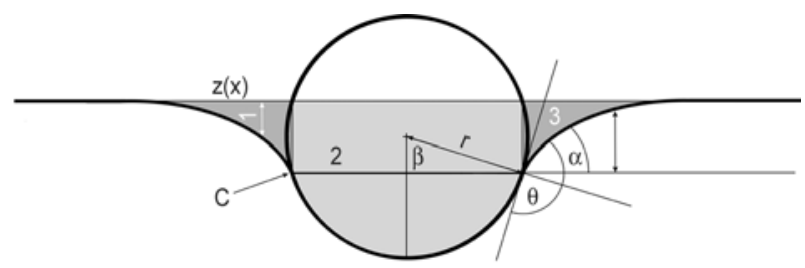

Figure 9. A liquid surface is deformed owing to a partly submerged cylinder. Solid, liquid and gas meet in a contact line $C$. Cylinder has the radius $r$. Contact angle is denoted by $\theta$. The height of the curved liquid surface is described by the function $z(x)$. restricted by the same vertical walls, the immersed cylinder surface from the bottom and by the water line from above.

Keller [18] showed that the surface tension force $f_{\mathrm{T}}$ is equal to the vertical component of the liquid surface tension multiplied by the total length of the two contact lines as well as being equal to the weight of the liquid displaced by the meniscus inside compartments 1 and 3 .

The angle $\alpha$ contained by a horizontal line and the liquid surface at the contact line $C$, see Figure 9, can be expressed using $\tan \alpha_{\mathrm{C}}=z^{\prime}\left(x_{\mathrm{C}}\right)$ where $z^{\prime}\left(x_{\mathrm{C}}\right)$ is the $x$ derivative of the liquid-gas interface displacement $z(x)$ at the contact line. The interface $z(x)$ between a liquid and a gas at rest satisfies the Laplace-Young equation (Equation (1)):

$\rho g z=\gamma \frac{z^{\prime \prime}}{\left(1+z^{\prime 2}\right)^{3 / 2}}$

where $\rho$ is the liquid density and $g$ denotes the acceleration due to gravity. The differential operator identity $\frac{\mathrm{d}}{\mathrm{d} x}=\frac{\mathrm{d} z}{\mathrm{~d} x} \frac{\mathrm{d}}{\mathrm{d} z}$ allows us to write a second $x$ derivative of $z$ as $z^{\prime \prime}=z^{\prime} \frac{\mathrm{d} z^{\prime}}{\mathrm{d} z}=\frac{1}{2} \frac{\mathrm{d}}{\mathrm{d} z} z^{\prime 2}$. Introducing the capillary constant, $a=\sqrt{2 \gamma /(\rho g)}$, one obtains the first integral of the Relation (1) in the form (Equation (2)):

$$
\int \frac{2 z}{a^{2}} \mathrm{~d} z=\frac{1}{2} \int \frac{\mathrm{d} z^{\prime 2}}{\left(1+z^{\prime 2}\right)^{3 / 2}}
$$

The relation $z^{\prime}=\sqrt{\frac{a^{4}}{\left(A a^{2}-z^{2}\right)^{2}}-1}$ between the tangent of the liquid meniscus slope $z^{\prime}(x)=\tan \alpha$ and its displacement $z(x)$ is derived from the integration of Equation (2) as shown in [19]. Conditions at infinity, $z=z^{\prime}=0$ for $x \rightarrow \infty$, result in $A=1$. Dimensionless displacement $Z=z / a$, introduced here for the sake of brevity, allows us to express the last relation in a comprehensive manner (Equation (3)):

$\tan \alpha=z^{\prime}=a \frac{\mathrm{d} Z}{\mathrm{~d} x}= \pm Z \frac{\sqrt{2-Z^{2}}}{1-Z^{2}}$

Integration of Equation (3) gives the liquid surface profile curve (Equation (4)):

$\frac{x-x_{0}}{a}=\sqrt{2-Z^{2}}+\frac{1}{\sqrt{2}} \ln \frac{Z}{2\left(\sqrt{2}+\sqrt{2-Z^{2}}\right)}$ 


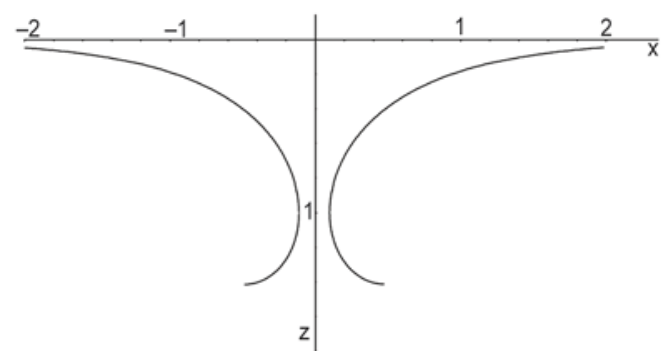

Figure 10. The shape of the liquid surface meniscus is plotted using inversion function $x(z)$ obtained from Equation (4)

where $x 0$ is an integration constant. The shape of the liquid surface meniscus obtained from Relation (4) is shown in Figure 10.

Total lift force due to surface tension $f_{\mathrm{T}}$ is also given here, for the sake of brevity, in its dimensionless form $F_{\mathrm{T}}$ being normalized using the product of the liquid surface tension $\gamma$ and the cylinder length $l$, that is, $F_{\mathrm{T}}=f_{\mathrm{T}} /(\gamma l)$. For $F_{\mathrm{T}}$ holds (Equation (5)):

$F_{\mathrm{T}}=2 \sin \alpha=\frac{2 z^{\prime}}{\sqrt{1+z^{\prime 2}}}=2 Z \sqrt{2-Z^{2}}$

The pressure force $f_{\mathrm{P}}$ equals the weight of the liquid which would fill compartment 2 . Similarly to the introduction of $F_{\mathrm{T}}$, the dimensionless pressure force $F_{\mathrm{P}}=f_{\mathrm{P}} /(\gamma l)$ equals to $\rho g$ multiplied by the area of compartment 2, see Figure 9. The last compartment comprises a rectangle and a circular segment. The rectangle has an area $S_{\mathrm{O}}=2 r \sin \beta \cdot z$, while the circular section area is $S_{\mathrm{S}}=r^{2}(\beta-\cos \beta \sin \beta)=$ $r^{2}\left[\beta-\frac{1}{2} \sin (2 \beta)\right]$. For the angle $\beta$ takes force $\beta=$ $\pi-\theta+\arctan \left(z^{\prime}\right)$. Therefore the dimensionless pressure force $F_{\mathrm{P}}$ can be written in a comprehensive manner using the capillary length a and the dimensionless radius $R=r / a$ in the following manner (Equation (6)):

$F_{\mathrm{P}}=\mathrm{R}^{2}\left[\beta-\frac{1}{2} \sin (2 \beta)\right]+2 R Z \sin \beta$

For the total dimensionless lift force $F_{\mathrm{L}}=F_{\mathrm{T}}+F_{\mathrm{P}}$ holds (Equation (7)):

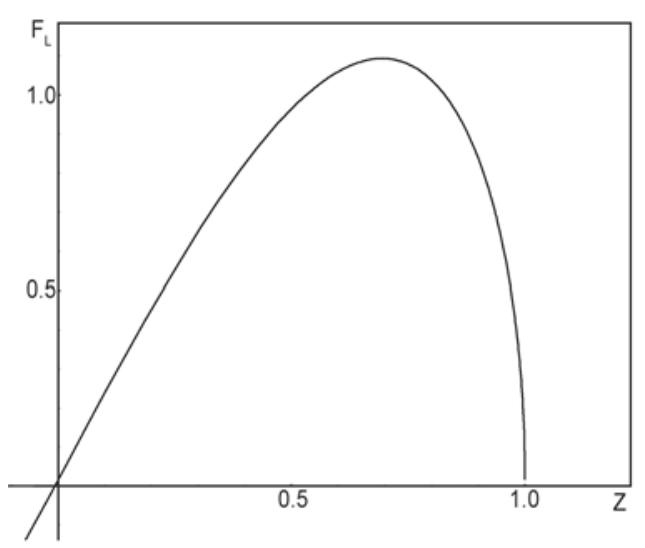

Figure 11. The relationship between $F_{\mathrm{L}}$ and $Z$ for $R=0.1$ and $\theta=1.5$

where has to be substituted from (3). The relationship between $F_{\mathrm{L}}$ and $Z$ for $R=0.1$ and $\theta=1.5$ is plotted in Figure 11.

Water surface tension against air at $25^{\circ} \mathrm{C}$ is $\gamma_{\text {water }}=$ $71.97 \mathrm{mN} / \mathrm{m}$ and its density is $\rho=997.0479 \mathrm{~kg} / \mathrm{m}^{3}$. From that follows the water capillary length $a_{\text {water }}=$ $3.837 \mathrm{~mm}$. Ethanol at $20^{\circ} \mathrm{C}$ against air has the surface tension $\gamma_{\text {ethanol }}=22.27 \mathrm{mN} / \mathrm{m}$, its density is $789.45 \mathrm{~kg} / \mathrm{m}^{3}$ and its capillary length is $a_{\text {ethanol }}=$ $2.399 \mathrm{~mm}$. Diameters of nanofibrous materials in this work are below $5 \cdot 10^{-6} \mathrm{~m}$. Therefore their dimensionless radii, $R=r / a$, are small, $R \leq 6.515 \cdot 10^{-4}$ for water and $2.084 \cdot 10^{-3}$ for ethanol.

Assuming that a cylindrical fiber is long, its radius is around $1 \mu \mathrm{m}$ and the contact angle $\theta$ is less than $90^{\circ}$, then the condition for the equilibrium between maximum lift force $f_{\mathrm{L}}$ and the cylinder weight $w$ is (Equation (8)):

$2 \gamma L \sin \theta+\pi r^{2} \rho L g=\pi r^{2} \rho_{\mathrm{S}} L g$

where $\rho_{\mathrm{S}}$ denotes the fiber mass density. From Relation (8) one derives the dimensionless relationship between the contact angle $\theta$, the dimensionless fiber radius $R$ and the ratio of densities $\rho_{\mathrm{S}} / \rho$ (Equation (9)).

$\theta=\arcsin \left[\pi R^{2}\left(\frac{\rho_{\mathrm{S}}}{\rho}-1\right)\right]$

$F_{\mathrm{L}}=2 Z \sqrt{2-Z^{2}}+R^{2}\left\{\pi-\theta+\arctan \left(z^{\prime}\right)-\frac{1}{2} \sin \left[2 \pi-2 \theta+2 \arctan \left(z^{\prime}\right)\right]\right\}+2 R Z \sin \left[\pi-\theta+\arctan \left(z^{\prime}\right)\right]$ 
Substituting the following values: $r=5 \cdot 10^{-6} \mathrm{~m}, a=$ $3.837 \cdot 10^{-3} \mathrm{~m}, \rho=1000 \mathrm{~kg} / \mathrm{m}^{3}$ and $\rho_{\mathrm{S}}=1145 \mathrm{~kg} / \mathrm{m}^{3}$, one arrives at the contact angle $\theta$ value to immerse the cylindrical fiber that is negligibly small, that is. One concludes the analysis of the problem with a final statement, that complete submersion and fiber mass density greater than the mass density of liquid are two necessary conditions for immersion of a micro or nanofibers.

The contact angles of the solutions used as liquid collectors onto a PCL membrane were measured experimentally and found to support the above introduced theoretical condition for fiber immersion into a liquid (see Figure 12). The membrane made from four conventionally electrospun PCLs is produced by hot press at temperature $60^{\circ} \mathrm{C}$ and loading $3,5 \mathrm{kPa}$ nanofibrous layers. The membrane's surface density is $24 \mathrm{~g} \cdot \mathrm{m}^{-2}$.

The contact angle measurements proved that fibers immersing into the liquid collector have to have zero contact angle, which confirms the theoretical explanation written in this chapter. The results shown in Figure 12 showed the contact angle of a drop on the PCL membrane is zero for water/ethanol mixtures $(6: 4 ; 5: 5 ; 4: 6 ; 3: 7 ; 2: 8 ; 1: 9 ; 0: 10)$. PCL fibers were mainly found to collect during wet electrospinning on the liquid collector surface where there were higher contact angles (see Figure 6).

\section{Conclusions}

Wet electrospun material morphologies are compared with those obtained by common electrospinning that employs a metallic collector. The comparison of internal morphologies of dry and wet electrospun materials shows that PCL beads appearing in dry spun fibers are generated by a fraction of thicker fibers developed during electrospinning process. These thicker fibers were generated in the whipping zone of a jet that has been trapped using instantaneous solidification caused by immersion of the jet into the non-solvent of the liquid collector. A very important phenomenon which was found during these experiments was the relatively slow dynamics of the creation of big PCL droplets between nanofibers. These droplets are probably formed due to the 'slow' Plateau-Rayleigh instability that occurs after the not-completely-solidified fiber is captured on the solid collector. The theoretical explanation of

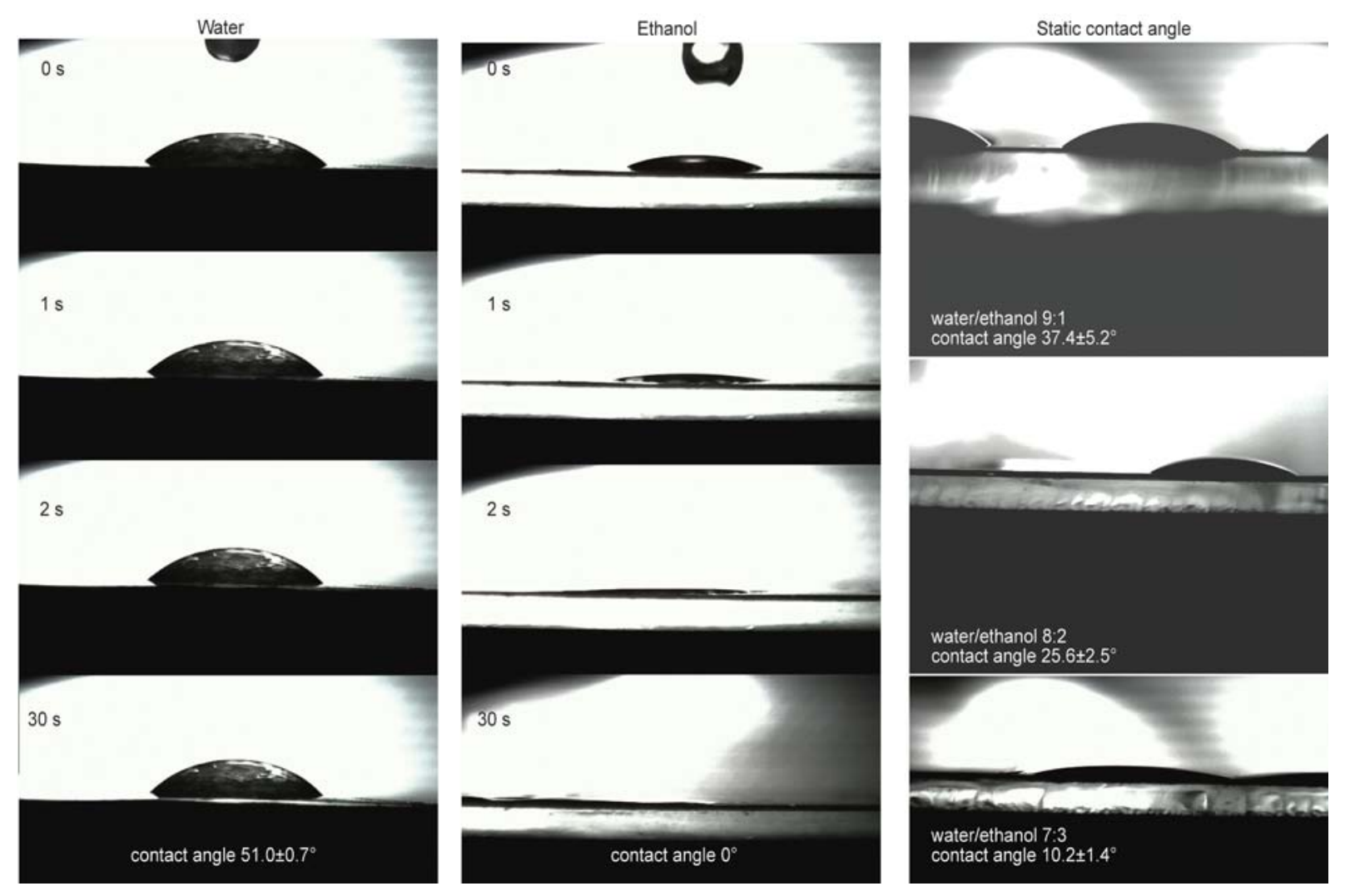

Figure 12. Photos of distilled water drops and water/ethanol mixture $(9: 1 ; 8: 2 ; 7: 3)$ drops placed onto a membrane made from PCL that are used for contact angle measurements. The static contact angles are measured 30 seconds after a drop impacts onto the membrane surface. For other water ethanol mixtures the resultant contact angles are the same as for pure ethanol drops. A drop falls down and disappears leaving a trace with zero contact angle. 
spontaneous immersion of a cylindrical fiber into a liquid is also introduced here.

The main result of the study is the occurrence of the dual fibrous structures composed of microfibers and nanofibers distributed uniformly in all samples with a three-dimensional structure produced from PCL by wet electrospinning into ethanol/distilled water mixture. Such materials can be used as a scaffold for tissue engineering. The fibers of bigger diameter have better mechanical properties and a larger pore size is important for three-dimensional cell distribution. Fibers of smaller diameter ensure good cell adhesion inside the scaffold. The optimal material electrospun into the liquid reservoir water/ ethanol with weight ratio 1:9 for its three-dimensional structure and high porosity and with almost no structural defects was chosen as the best material for further in-vitro tests as a scaffold for tissue engineering.

\section{Acknowledgements}

The support for this research was provided by The Ministry of Interior of the Czech Republic, program BV II/2-VS, grant VG20102014049, 'Nanomaterials for personal protection against CBRN substances'. The paper was supported in part by the Project OP VaVpI Centre for Nanomaterials, Advanced Technologies and Innovation CZ.1.05/2.1.00/ 01.0005. D.L. acknowledges the support of GACR, Grant no. P208/12/0105. Authors thank to Jana Privratska for discussion about the mathematical derivations, Marcela Cudlinova for her help with Figure 4, Petr Mikes for his help with Figure 1; 10 and 11 and Jiri Militky for discussion about wet spinning technologies (all from Technical University of Liberec).

\section{References}

[1] Yokoyama Y., Hattori S., Yoshikawa C., Yasuda Y., Koyama H., Takato T., Kobayashi H.: Novel wet electrospinning system for fabrication of spongiform nanofiber 3-dimensional fabric. Materials Letters, 63, 754756 (2009). DOI: 10.1016/j.matlet.2008.12.042

[2] Ali A. A., Al-Asmari A. Kh.: Wet-electrospun CuNP/ carbon nanofibril composites: Potential application for micro surface-mounted components. Applied Nanoscience, 2, 55-61 (2012). DOI: $10.1007 / \mathrm{s} 13204-011-0042-\mathrm{Z}$

[3] Lukáš D., Sarkar A., Martinová L., Vodsed’álková K., Lubasová D., Chaloupek J., Pokorný P., Mikeš P., Chvojka J., Komárek M.: Physical principles of electrospinning (electrospinning as a nano-scale technology of the twenty-first century). Textile Progress, 41, 59-140 (2009).

DOI: $\underline{10.1080 / 00405160902904641}$
[4] Zhong S., Zhang Y., Lim C. T.: Fabrication of large pores in electrospun nanofibrous scaffolds for cellular infiltration: A review. Tissue Engineering Part B: Reviews, 18, 77-87 (2012). DOI: $10.1089 /$ ten.TEB.2011.0390

[5] Quan S-L., Kang S-G., Chin I-J.: Characterization of cellulose fibers electrospun using ionic liquid. Cellulose, 17, 223-230 (2010). DOI: $10.1007 / \mathrm{s} 10570-009-9386-\mathrm{x}$

[6] Barber P. S., Griggs C. S., Bonner J. R., Rogers D.: Electrospinning of chitin nanofibers directly from an ionic liquid extract of shrimp shells. Green Chemistry, 15, 601-607 (2013). DOI: $10.1039 / \mathrm{c} 2 \mathrm{gc} 36582 \mathrm{k}$

[7] Shin T. J., Park S. Y., Kim H. J., Lee H. J., Youk J. H.: Development of 3-D poly(trimethylenecarbonate-co$\varepsilon$-caprolactone)-block-poly( $p$-dioxanone) scaffold for bone regeneration with high porosity using a wet electrospinning method. Biotechnology Letters, 32, 877882 (2010). DOI: $10.1007 / \mathrm{s} 10529-010-0235-7$

[8] Eberli D.: Tissue engineering. InTech, Rijeka (2010).

[9] Reneker D. H., Yarin A. L.: Electrospinning jets and polymer nanofibers. Polymer, 49, 2387-2425 (2008). DOI: $10.1016 /$ j.polymer.2008.02.002

[10] Fang J., Wang H., Niu H., Lin T., Wang X.: Evolution of fiber morphology during electrospinning. Journal of Applied Polymer Science, 118, 2553-2561 (2010). DOI: 10.1002/app.32569

[11] Gupta V. B., Kothari V. K.: Manufactured fiber technology. Chapman and Hall, London (1997).

[12] Takajima T.: Advanced fiber spinning technology. Woodhead Publishing Limited, Cambridge (1994).

[13] Walczak Z. K.: Processes of fiber formation. Elsevier, Amsterdam (2002).

[14] Sattler R., Gier S., Eggers J., Wagner C.: The final stages of capillary break-up of polymer solutions. Physics of Fluids, 24, 023101/1-023101/21 (2012). DOI: $10.1063 / 1.3684750$

[15] Brown P. J., Stevens K.: Nanofibers and nanotechnology in textiles. Woodhead, Cambridge (2007).

[16] Yildirim E. D.: Plasma and protein surface functionalization for three-dimensional polycaprolactone tissue scaffolds. PhD Thesis, Drexler University, College of Engineering (2010).

[17] Vella D., Mahdevan L.: The 'Cheerios effect'. American Journal of Physics, 73, 817-825 (2005). DOI: $10.1119 / 1.1898523$

[18] Keller J. B.: Surface tension force on a partly submerged body. Physics of Fluids, 10, 3009-3010 (1998). DOI: $10.1063 / 1.869820$

[19] Landau L. D., Lifshitz E. M.: Course of theoretical physics, Volume 6: Fluid mechanics. Pergamon Press, Oxford (1959). 